\title{
Nedaplatin as a Single-Agent Chemotherapy May Support Palliative Therapy for Patients with Adenoid Cystic Carcinoma: A Case Report
}

\author{
Hiroyuki Hirakawa $^{a}$ Takayoshi Kiba $^{\text {b-d }}$ Yuko Saito $^{a}$ \\ Yoshiteru Watanabe ${ }^{a}$ Takahiro Suzuki ${ }^{\mathrm{e}}$ Nobuo Ota ${ }^{\mathrm{e}}$ \\ a Department of Pharmacy, Tohoku Medical and Pharmaceutical University Hospital, \\ Sendai, Japan; ${ }^{b}$ Department of Clinical Oncology, Tohoku Medical and Pharmaceutical \\ University Hospital, Sendai, Japan; 'Department of Life Sciences, Faculty of Science, \\ Okayama University of Science, Okayama, Japan; ${ }^{\mathrm{d} D i v i s i o n}$ of Modern Medical \\ Technology, Institute for Clinical Research, National Hospital Organization Kure \\ Medical Center, Kure, Japan; ${ }^{e}$ Department of Otorhinolaryngology, Tohoku Medical and \\ Pharmaceutical University Hospital, Sendai, Japan
}

\section{Keywords}

Nedaplatin · Adenoid cystic carcinoma - Submandibular gland - Lactate dehydrogenase - Case report

\section{Abstract}

Adenoid cystic carcinoma (ACC) is a rare form of adenocarcinoma, which is a broad term describing any cancer that begins in the glandular tissues. It can be found in the head and neck. We report a patient with recurrent ACC arising from the submandibular gland, treated with $100 \mathrm{mg} / \mathrm{m}^{2}$ nedaplatin every 4 weeks. Although our patient's lactate dehydrogenase 


\section{Case Reports in Oncology}

levels, which is produced by ACC, showed a rising trend throughout the treatment, the level decreased for approximately 2 weeks immediately after administration of nedaplatin every 4 weeks. Thus, there is a possibility that the agent may be effective. Complications such as anorexia and nausea were observed, but they were tolerated and manageable. Nedaplatin may be considered as a supportive agent during palliative therapy for patients with ACC. More clinical trials regarding nedaplatin are necessary, as this study may indicate that a medical approach works well for ACC.

(C) 2017 The Author(s)

Published by S. Karger AG, Basel

\section{Introduction}

Adenoid cystic carcinoma (ACC) is a rare form of malignant neoplasm that most commonly develops in the salivary glands or other regions of the head and neck. In some cases, ACC may arise in other primary sites, such as the skin, the breast, the head of the cervix in women, the prostate gland in men, or other areas. ACC is common in middle-aged adults, and is rare in people younger than 20 years of age. Some investigators have suggested that there appears to be a slight female preponderance, and this gender-specific trend could be due to the incidence rate in female organs [1]. ACC tends to spread along nerves, known as a perineural invasion, or through the bloodstream, lymph system, or other means. It spreads to the lymph nodes in only about $5-10 \%$ of all cases (http://www.cancer.net/cancertypes/adenoid-cystic-carcinoma/overview). The most common sites of metastatic spread of ACC are the lungs, liver, and bones.

Standard treatment includes complete surgical excision and additional radiation therapy in selected patients with a positive surgical margin, local recurrence, or high-grade malignancy. Systemic chemotherapy, based on cisplatin or cisplatin in combination with other agents including doxorubicin, vinorelbine, and fluorouracil has benefited certain patients with advanced disease caused by recurrent, metastatic, and unresectable tumors. It was reported that cisplatin monotherapy achieved a complete response (CR) [2]. However, there are no reports on nedaplatin yet, which has similar characteristics to cisplatin.

Nedaplatin (cis-diammine-glycolatoplatinum) is a second cisplatin analog and was developed in 1983 by Shionogi \& Co., Ltd., Osaka, Japan, to provide treatment with an effectiveness similar to that of cisplatin (http://www.e-search.ne.jp/ jpr/PDF/SHIONO00.PDF). It is used for treating head and neck cancer, small cell lung cancer, non-small cell lung cancer, esophageal cancer, bladder cancer, testicular tumor, ovarian cancer, and uterine cervical cancer. Cisplatin often induces nephrotoxicity and gastrointentinal toxicity, which limit its clinical use. Nedaplatin is an agent that demonstrated less of these toxicities [3]. According to the National Comprehensive Cancer Network guidelines, cisplatin is associated with a high risk for emesis ( $90 \%$ frequency of emesis), but nedaplatin is not categorized. In the clinical practice guidelines of the Japan Society of Clinical Oncology, it is categorized as moderate emesis risk (30-90\% frequency of emesis). The dose-limiting toxicity of nedaplatin is characterized by thrombocytopenia. Several phase II studies have demonstrated the efficacy of nedaplatin combination therapy in head and neck cancer [4-6]. Moreover, a recent phase III study indicated that nedaplatin plus docetaxel was superior to cisplatin plus docetaxel with regard to overall survival in advanced or relapsed squamous cell lung cancer 


\section{Case Reports in Oncology}

patients [7]. Based on this evidence, nedaplatin is currently the third most commonly used platinum agent, with only cisplatin and carboplatin being used more frequently. This case report shows the therapeutic efficacy of nedaplatin and reports the results obtained during the treatment of ACC.

\section{Case Presentation}

A 43-year-old woman was referred to the Tohoku Medical and Pharmaceutical University Hospital with ACC arising from the right submandibular gland. At another hospital, she had already received chemotherapy with co-administration of 5 -fluoruracil and cisplatin. The dosages of cisplatin and 5 -fluoruracil were $80 \mathrm{mg} / \mathrm{m}^{2}$ (day 1) and 1,000 mg/m $/ \mathrm{m}^{2}$ (days 1-5), respectively. She had received this regimen for 2 courses, but the effect measurement was progressive disease (PD). The second therapy had been S1 (tegafur, gimeracil, oteracil potassium). The dosage of S1 was $120 \mathrm{mg} /$ body. She had received S1 therapy for 2 months, but her primary lesion progressed, and the lung lesion was stable (stable disease, SD). For her primary lesion, she had received cyberknife 35 Gy for 10 days. This therapy was effective for the primary lesion, but thereafter, the lung metastatic lesions were PD.

Thereafter, she was referred to our hospital. She had a weekly chemotherapy with a combination of paclitaxel and cetuximab. Initially, $80 \mathrm{mg} / \mathrm{m}^{2}$ paclitaxel was administered for 3 weeks, together with $400 \mathrm{mg} / \mathrm{m}^{2}$ cetuximab per week. Then, she received $80 \mathrm{mg} / \mathrm{m}^{2}$ paclitaxel weekly plus $250 \mathrm{mg} / \mathrm{m}^{2}$ cetuximab from the next week. After 3 courses, she had a CT examination, showing that the lung lesions were PD. Her lungs also appeared to have malignant effusion, and she was thus treated with OK-432 injections for intrapleural therapy. Thereafter, she was selected for nedaplatin therapy. The dosage was $100 \mathrm{mg} / \mathrm{m}^{2}$ nedaplatin every 4 weeks.

Our patient was evaluated for toxicity using the Common Terminology Criteria for Adverse Events (CTCAE) version 4.0. (http://ctep.cancer.gov/protocolDevelopment/electronic_applications/ctc.htm\#ctc_40). On the third day of the first course, she had grade 2 anorexia and nausea. A dose of aprepitant, which addresses nausea, reduced her symptoms. Moreover, thrombocytopenia was not seen while she was administered nedaplatin. The CT examination before this first treatment with nedaplatin detected multiple lung metastases (Fig. 1). After the administration of 4 courses of nedaplatin, the number of lung metastases increased (Fig. 1). In addition, the CT examination after 5 courses of this treatment detected 2 brain metastases (approximately $1 \mathrm{~cm}$ in diameter each); therefore, she was further treated with gamma knife radiosurgery. Although her lactate dehydrogenase (LDH) levels, which is produced by ACC, showed a rising trend throughout the treatment, the level decreased for approximately 2 weeks immediately after the administration of nedaplatin every 4 weeks (Fig. 2). Most of her LDH types were LDH2, and the ratio was $36 \%$. This indicates a possibility that the treatment may be effective. 


\section{Case Reports in Oncology}

\section{Discussion}

Here, we report on a patient treated with nedaplatin for ACC arising from a submandibular gland. Effective agents are rare, and new chemotherapy agents are under investigation. Cisplatin is the most commonly reported agent in monotherapy. Licitra et al. [2] reported that cisplatin for the treatment of ACC achieved CR in 15\%, SD in 46\%, and PD in 38\% of their population. Moreover, typical studies with single-agent chemotherapy for ACC are summarized in Table 1 [2, 8-12]. However, the efficacy of nedaplatin for ACC has yet to be established.

Nedaplatin has been used extensively to treat patients with non-small cell lung cancer. There are some studies that compared the survival benefits of nedaplatin and cisplatin in the treatment of non-small cell lung cancer. Shan et al. [13] reported that the mean chemotherapy duration was 3.3 cycles in the cisplatin group and 4.1 cycles in the nedaplatin group. The median survival time was 15 months in the cisplatin group, and 20 months in the nedaplatin group. The 1-, 2-, and 3-year overall survival rates were 62.4, 25.7, and 15.8\% and 78.9, 38.9, and $16.8 \%$ in the cisplatin and nedaplatin groups, respectively. The incidence rate of nausea/vomiting, anorexia, and weight loss was higher in the cisplatin group compared to the nedaplatin group ( 36.1 vs. $8.4 \%, 17.3$ vs. $5.8 \%$, and 9.9 vs. $1 \%$, respectively). In many cases, the administration of nedaplatin may not show a marked response; however, overall survival may be prolonged. Because patients receiving nedaplatin experience less toxicity and show good compliance with the regimen, these patients can complete more cycles of therapy. Shan et al. [13] also reported that in non-small cell lung cancer patients, more chemotherapy cycles can reduce the mortality risk by $46 \%$.

On the other hand, the LDH level decreases after the administration of nedaplatin. It is known that LDH splits up into types LDH1 to LDH5. In particular, cancer cells result in elevated LDH2 levels. Our patient's LDH was mostly LDH2. Although her LDH levels, which is produced by ACC, showed a rising trend throughout the treatment, the level decreased for approximately 2 weeks immediately after the administration of nedaplatin every 4 weeks. Yuh and Kim [14] suggested that LDH2 is the most commonly elevated LDH type in nonsmall cell lung cancer patients. Serum LDH levels following chemotherapy are associated with the response to chemotherapy. Furthermore, Faloppi et al. [15] suggested that LDH seemed able to predict clinical outcome in terms of progression-free survival and overall survival for hepatocellular carcinoma patients treated with sorafenib. In our case, because our patient's LDH level decreased over a brief period immediately after the administration of nedaplatin every 4 weeks, it is possible that this regimen may be effective.

Side effects such as anorexia and nausea were observed, although they were well tolerated and manageable. Our patient had grade 2 anorexia and nausea, but the oral administration of aprepitant relieved some of these symptoms. Thrombocytopenia was not observed. Reports suggested that nedaplatin therapy seemed to be associated with acceptable and manageable side effects. 


\section{Case Reports in Oncology}

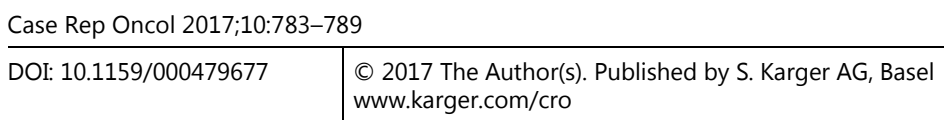

Hirakawa et al.: Nedaplatin as a Single-Agent Chemotherapy May Support Palliative

Therapy for Patients with Adenoid Cystic Carcinoma: A Case Report

\section{Conclusion}

Further clinical trials regarding nedaplatin are needed in order to evaluate the impact of this novel agent used here and to make a long-term prognosis in patients with ACC.

\section{Statement of Ethics}

The authors have no ethical conflict to disclose.

\section{Disclosure Statement}

The authors declare that there is no conflict of interest regarding the publication of this article.

\section{References}

Jaso J, Malhotra R: Adenoid cystic carcinoma. Arch Pathol Lab Med 2011;135:511-515.

Licitra L, Marchini S, Spinazzè S, et al: Cisplatin in advanced salivary gland carcinoma. A phase II study of 25 patients. Cancer 1991;68:1874-1877.

3 Shimada M, Itamochi H, Kigawa J: Nedaplatin: a cisplatin derivative in cancer chemotherapy. Cancer Manag Res 2013;5:67-76.

4 Kurita H, Yamamoto E, Nozaki S, Wada S, Furuta I, Miyata M, Kurashina K: Multicenter phase 2 study of induction chemotherapy with docetaxel and nedaplatin for oral squamous cell carcinoma. Cancer Chemother Pharmacol 2010;65:503-508.

5 Ohashi T, Ohnishi M, Tanahashi S, Murai M: Efficacy and toxicity of concurrent chemoradiotherapy with nedaplatin and S-1 for head and neck cancer. Jpn J Clin Oncol 2011;41:348-352.

6 Hoshikawa H, Kishino T, Mori T, et al: Clinical outcomes of nedaplatin and S-1 treatment with concurrent radiotherapy in advanced head and neck cancer. Acta Otolaryngol 2015;135:103-108. Shukuya T, Yamanaka T, Seto T, et al: Nedaplatin plus docetaxel versus cisplatin plus docetaxel for advanced or relapsed squamous cell carcinoma of the lung (WJOG5208L): a randomised, open-label, phase 3 trial. Lancet Oncol 2015;16:1630-1638. Tannock IF, Sutherland DJ: Chemotherapy for adenocystic carcinoma. Cancer 1980;46:452-454. Verweij J, de Mulder PH, de Graeff A, et al: Phase II study on mitoxantrone in adenoid cystic carcinomas of the head and neck. EORTC Head and Neck Cancer Cooperative Group. Ann Oncol 1996;7:867-869.

10 Airoldi M, Pedani F, Succo G, et al: Phase II randomized trial comparing vinorelbine versus vinorelbine plus cisplatin in patients with recurrent salivary gland malignancies. Cancer 2001;91:541-547.

11 Vermorken JB, Verweij J, de Mulder PH, et al: Epirubicin in patients with advanced or recurrent adenoid cystic carcinoma of the head and neck: a phase II study of the EORTC Head and Neck Cancer Cooperative Group. Ann Oncol 1993;4:785-788.

12 Gilbert J, Li Y, Pinto HA, Jennings T, Kies MS, Silverman P, Forastiere AA: Phase II trial of taxol in salivary gland malignancies (E1394): a trial of the Eastern Cooperative Oncology Group. Head Neck 2006;28:197-204.

13 Shan J, Xiong Y, Wang D, et al: Nedaplatin- versus cisplatin-based chemotherapy in the survival time of patients with non-small cell lung cancer. Mol Clin Oncol 2015;3:543-549.

-14 Yuh YJ, Kim SR: Acute dehydrogenase (LDH) as a tumor marker for non-small cell lung cancer. Cancer Res Treat 2002;34:339-344.

15 Faloppi L, Scartozzi M, Bianconi M, et al: The role of LDH serum levels in predicting global outcome in HCC patients treated with sorafenib: implications for clinical management. BMC Cancer 2014;14:110. 


\section{Case Reports in Oncology}
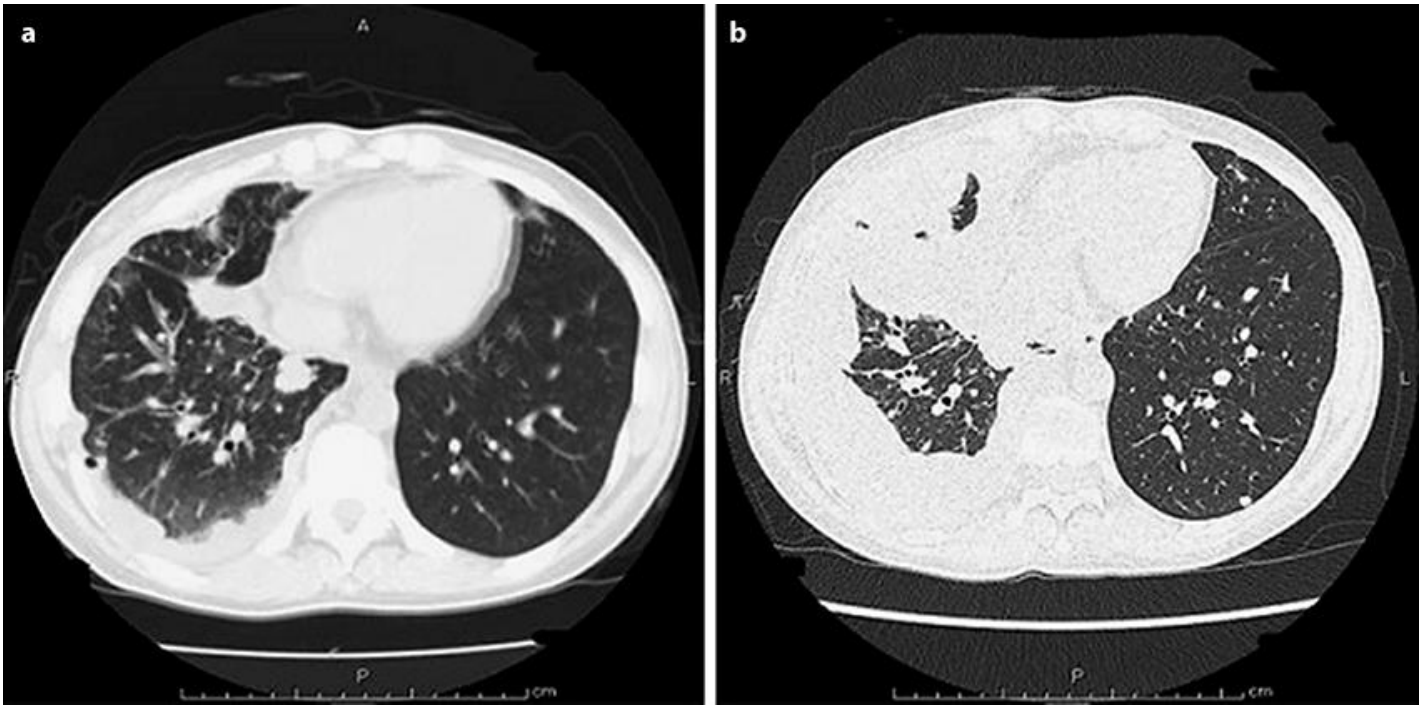

Fig. 1. CT scan: lung metastasis before (a) and after 4 courses of nedaplatin treatment (b).

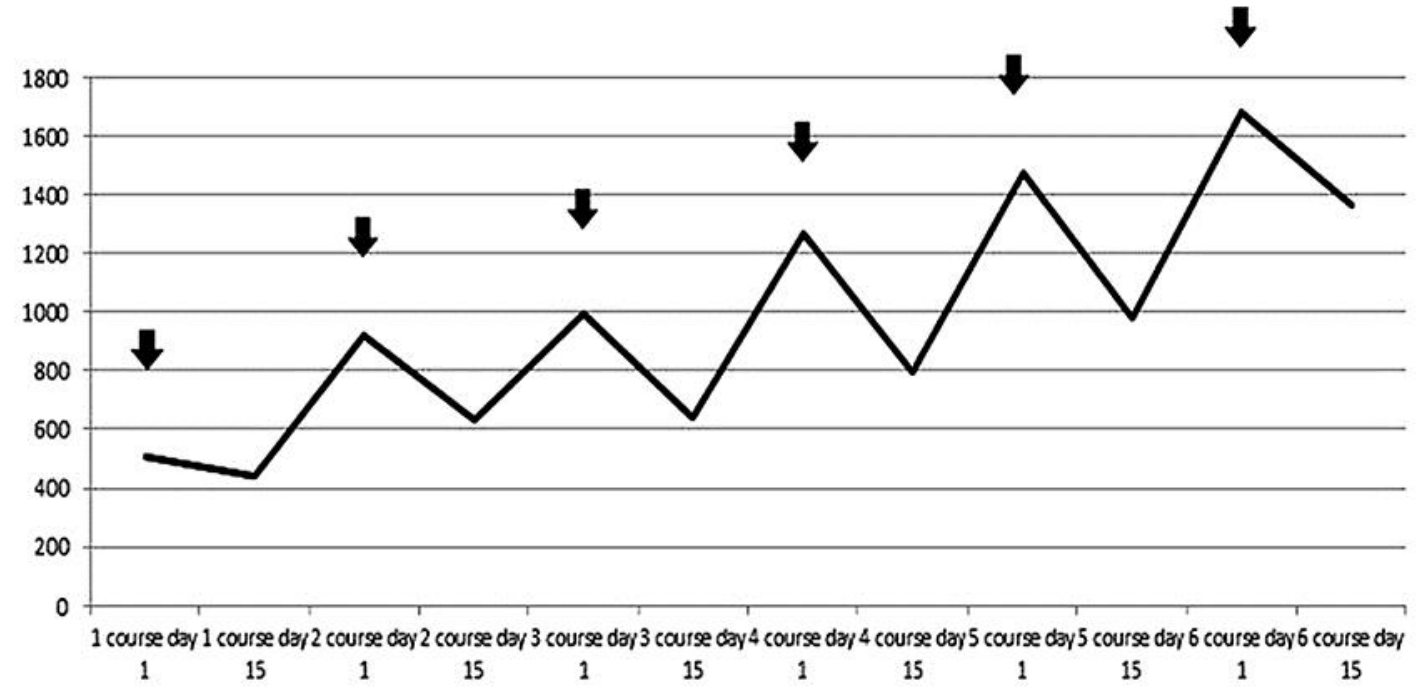

Fig. 2. LDH curves in our ACC patient treated with nedaplatin. Black arrows mean that the patient received nedaplatin therapy. 


\section{Case Reports in Oncology}

\begin{tabular}{l|l} 
DOI: $10.1159 / 000479677$ & (C) 2017 The Author(s). Published by S. Karger AG, Basel
\end{tabular} www.karger.com/cro

Hirakawa et al.: Nedaplatin as a Single-Agent Chemotherapy May Support Palliative

Therapy for Patients with Adenoid Cystic Carcinoma: A Case Report

Table 1. Outcomes of monotherapy in adenoid cystic carcinoma

\begin{tabular}{|c|c|c|c|c|c|}
\hline Author [Ref.] & Agent & Patients, $n$ & $\mathrm{CR}+\mathrm{PR}$ & SD & $\mathrm{PD}$ \\
\hline Licitra et al. [2] & cisplatin & 13 & 2 & 6 & 5 \\
\hline Tannock and Sutherland [8] & 5-fluorouracil & 12 & 4 & 2 & 9 \\
\hline Tannock and Sutherland [8] & methotrexate & 7 & 0 & 0 & 7 \\
\hline Tannock and Sutherland [8] & cyclophosphamide & 3 & 0 & 0 & 3 \\
\hline Tannock and Sutherland [8] & chloroambucil & 2 & 0 & 0 & 2 \\
\hline Tannock and Sutherland [8] & vincristine & 3 & 0 & 1 & 2 \\
\hline Verweij et al. [9] & mitoxantrone & 32 & 4 & 22 & 6 \\
\hline Airoldi et al. [10] & vinorelbine & 13 & 4 & \multicolumn{2}{|c|}{9} \\
\hline Vermorken et al. [11] & epinephrine & 20 & 2 & \multicolumn{2}{|c|}{18} \\
\hline Gilbert et al. [12] & paclitaxel & 13 & 0 & 7 & 6 \\
\hline
\end{tabular}

$\mathrm{CR}$, complete response; PR, partial response; SD, stable disease; PD, progressive disease. 